\title{
Activation of Insect Phenoloxidase after Injury: Endogenous versus Foreign Elicitors
}

\author{
Gawa Bidla $^{a}$ Thomas Hauling ${ }^{a}$ Mitchell S. Dushay ${ }^{b}$ Ulrich Theopold ${ }^{a}$ \\ a Department of Molecular Biology and Functional Genomics, University of Stockholm, Stockholm, and \\ ${ }^{b}$ Department of Comparative Physiology, EBC, Uppsala University, Uppsala, Sweden
}

\section{Key Words}

Coagulation $\cdot$ Comparative immunology $\cdot$ Drosophila melanogaster $\cdot$ Hemostasis $\cdot$ Innate immunity $\cdot$ Insects . Pattern recognition $\cdot$ Sepsis $\cdot$ Wound healing

\begin{abstract}
The enzyme phenoloxidase (PO) is one of the first immune molecules that was identified in invertebrates. Recently, the immune function of $\mathrm{PO}$ has been challenged. We tested how $\mathrm{PO}$ is activated following injury in 2 insects, i.e. the fruit fly Drosophila melanogaster and the wax moth Galleria mellonella. Rapid PO activation in Drosophila was limited to discrete areas of the hemolymph clot which forms after injury. Surprisingly, unlike systemic PO activation during bacterial sepsis, clot melanization was not sensitive to microbial elicitors in our assay. Instead, Drosophila clot melanization was activated by endogenous signals such as apoptotic cells and was superinduced by phosphatidylserine, a negatively charged phospholipid normally found on the inner surface of the plasma membrane and exposed during apoptosis. In contrast, melanization in G. mellonella hemolymph was stronger and more uniform and was sensitive to peptidoglycan. This shows that both exogenous and endogenous signals can trigger the same immune mechanism in speciesand context-dependent ways. Our findings have implications for the evolutionary dynamics of immune mechanisms and are in agreement with recent comparisons of insect immune transcriptomes.

Copyright $\odot 2008$ S. Karger AG, Basel
\end{abstract}

\section{Introduction}

One of the key immune reactions in invertebrates is the activation of tyrosinase-type phenoloxidases (POs; EC 1.10.3.1), which catalyze several reactions leading to the crosslinking of proteins, the production of reactive intermediates with potential cytotoxic activity [1] and ultimately to the production of melanin $[2,3]$. The proenzyme prophenoloxidase (proPO) can be activated upon contact with microbes as well as during the formation of capsules that surround larger foreign objects such as parasitoid eggs. In Drosophila melanogaster, full systemic activation of proPO during septic infections has been shown to depend on the activation of a proteolytic cascade and breakdown of the inhibitor serpin 27A (Spn27A) [4-8]. Both these events are induced after recognition of microbial products through pattern recognition receptors [in particular peptidoglycan (PGN) recognition proteins], leading to transcription of genes including regulators of proPO activation. This activation of $\mathrm{PO}$ during the insect immune response is consistent with the idea that microbial signals are key activators of immune reactions [9]. Nevertheless, the importance of proPO activation for insect immunity is controversial [2, 7, 8, 10-12].

$\mathrm{PO}$ is also involved in coagulation in insects. POs were identified in the clot in Galleria mellonella [13], in the Anopheles gambiae mosquito [14] and in Drosophila [15], where it was shown to contribute to clot hardening [10, 16]. While tyrosinase-type PO activity in the clot has a

\section{KARGER}

Fax +4161306 1234 E-Mail karger@karger.ch www.karger.com
Dr. Ulrich Theopold

Department of Molecular Biology and Functional Genomics

University of Stockholm

SE-10691 Stockholm (Sweden)

Tel. +46 816 4181, Fax +46815 2350, E-Mail uli@molbio.su.se 
physiological aspect similar to sclerotinization of the cuticle, it is noted that sclerotinization of the insect cuticle appears to require laccase-type POs (EC 1.10.3.2), rather than tyrosinases [17].

To identify activators of proPO and to learn more about the mode of proPO activation, we compared melanization in D. melanogaster and the wax moth G. mellonella, two established insect models for wound healing $[15,18,19]$. In both these insects, proPO is released from specialized hemocytes, called crystal cells and oenocytoids, respectively, within minutes after bleeding. We observed marked differences in the pattern of melanization. In Drosophila in vivo and ex vivo preparations, melanization was highly localized even in the presence of a bacterial elicitor (PGN). In contrast, Galleria larvae injected with PGN melanized systemically, and ex vivo clot preparations darkened more uniformly. We also found that Drosophila proPO could be superactivated by phosphatidylserine (PS), an endogenous elicitor, while PGN was more effective in Galleria. When infection-dependent breakdown of Drosophila Spn27A was mimicked using Spn27A mutants, the pattern of melanization resembled that in uninfected Galleria, suggesting that differences in the inhibition of proPO activation might explain some of our observations. We propose a model for proPO activation incorporating these findings.

\section{Materials and Methods}

\section{Insect Culture}

Flies were kept at $25^{\circ} \mathrm{C}$ on a 12 -hour light/dark cycle on potato sucrose medium. Washed 3rd-instar larvae of Drosophila were used. The Drosophila strains used were: $w^{1118}$ (wild type), spn27A ${ }^{1} / C y O$ actin-GFP [4], Hemese-Gal4 UAS-GFP.nls [20] and UAS-grim [21]. Gal4 > UAS experiments were performed at $25^{\circ} \mathrm{C}$. Galleria larvae were reared on a 12 -hour light/dark cycle at $25^{\circ} \mathrm{C}$ on an artificial diet.

\section{Histology}

The preparation of clot samples was essentially performed as previously described in the hanging drop assay [10]. Hemolymph from 5 Drosophila larvae and a comparable amount for Galleria were each incubated upside down for $30 \mathrm{~min}$ in a humid chamber at $25^{\circ} \mathrm{C}$. The formed clot was then caught on a coverslip, and images were taken with a Coolpix 4500 digital camera (Nikon) adapted to a Leitz Orthoplan microscope. The clot preparation shown in figures $4 \mathrm{~F}$ and $\mathrm{G}$ was reconstituted by incubating the clot on the coverslip for another $30 \mathrm{~min}$ with $20 \mathrm{mM}$ DL-3,4-dihydroxyphenylalanine (DOPA) in phosphate buffer ( $\mathrm{pH}$ 6.6).

PO Activation

Photometric measurement of hemolymph PO activity has recently been described [16]. L- $\alpha$-Phosphatidylethanolamine (PE)
(P8068), L- $\alpha$-phosphatidylinositol sodium (P0639), L- $\alpha$-phosphatidylcholine (PC) (P3556) and L- $\alpha$-phosphatidyl-L-serine (P0474) were purchased from Sigma. Each lipid was dissolved in buffer A (50 mM Tris, $0.1 \mathrm{M} \mathrm{NaCl}, \mathrm{pH}$ 7.5), which has been described elsewhere [22], and used at a final concentration of $10 \mu \mathrm{g} / \mathrm{ml}$. Lipopolysaccharide (LPS; Escherichia coli O55:B5, Sigma) and PGN (Bacillus megaterium Bm11, kindly provided by H. Steiner, Stockholm, Sweden) were similarly suspended.

By quickly bleeding 12 larvae of Drosophila each time into a well of a 15 -well slide and withdrawing $7 \mu \mathrm{l}$, this volume of hemolymph was pooled on ice in $50 \mu$ l of anticoagulant Ringer's solution containing $20 \mathrm{~mm}$ EDTA instead of $\mathrm{CaCl}_{2}$. After pipette mixing, $5 \mu \mathrm{l}$ of pooled hemolymph was aliquoted in triplicate or quadruplicate to $1 \mu \mathrm{l}$ of each phospholipid, PGN, LPS and buffer A (control) on ice. For Galleria, 4 drops of hemolymph from 4 thinstar larvae were pooled on ice in $2 \mathrm{ml}$ of Galleria Ringer's anticoagulant saline by amputating a pro-leg. Galleria hemolymph was then pipette-mixed, and triplicate $140-\mu$ l aliquots were transferred to $10 \mu \mathrm{l}$ of different elicitors on ice.

After samples were allowed to activate at $25^{\circ} \mathrm{C}$ for $10 \mathrm{~min}, 45$ and $150 \mu \mathrm{l}$ of DOPA $(20 \mathrm{~mm})$ were added to Drosophila and Galleria samples, respectively, and the optical density (OD) was read at $490 \mathrm{~nm}$ with a $\operatorname{Vmax}^{\mathrm{TM}}$ Kinetic Microplate Reader (Molecular Devices). The OD was measured again after incubating the samples for $30 \mathrm{~min}$ at $37^{\circ} \mathrm{C}$. Activation of $\mathrm{PO}$ was measured as the change in $\mathrm{OD}$. Photometric measurements were repeated at least 3 times, basically with the same result.

\section{PGN Injection}

Drosophila and Galleria last-instar larvae were injected with PGN from B. megaterium Bm11 (kindly provided by H. Steiner) suspended in distilled sterile water at a concentration of approximately $2.5 \mathrm{mg} / \mathrm{ml}$. Larvae were injected in the abdominal region using glass-capillary-drawn needles connected to a custom-made injector. Melanization was monitored over a period of $2 \mathrm{~h}$ in both Drosophila and Galleria. As a control, distilled sterile water was injected instead of PGN.

Statistical Analysis

Samples from the photometric measurements were compared using ANOVA (available at http://faculty.vassar.edu/lowry// anovalu.html) or a Student $t$ test (STT; Microsoft Excel). The samples shown in figure 3 were triplicates and differed significantly from the control at $<0.05$ (STT for Drosophila, fig. 3A) or $<0.01$ (STT for Galleria, fig. 3B, and Drosophila when analyzing quadruplicates).

\section{Results}

\section{Melanization Patterns in Bacterial \\ Elicitor-Challenged Galleria and Drosophila \\ Larvae Are Different}

To compare proPO activation between Galleria and Drosophila, we injected PGN into larvae from both species. Injection of PGN in Galleria led to melanization throughout the hemocoel (fig. 1A, B), while injected 

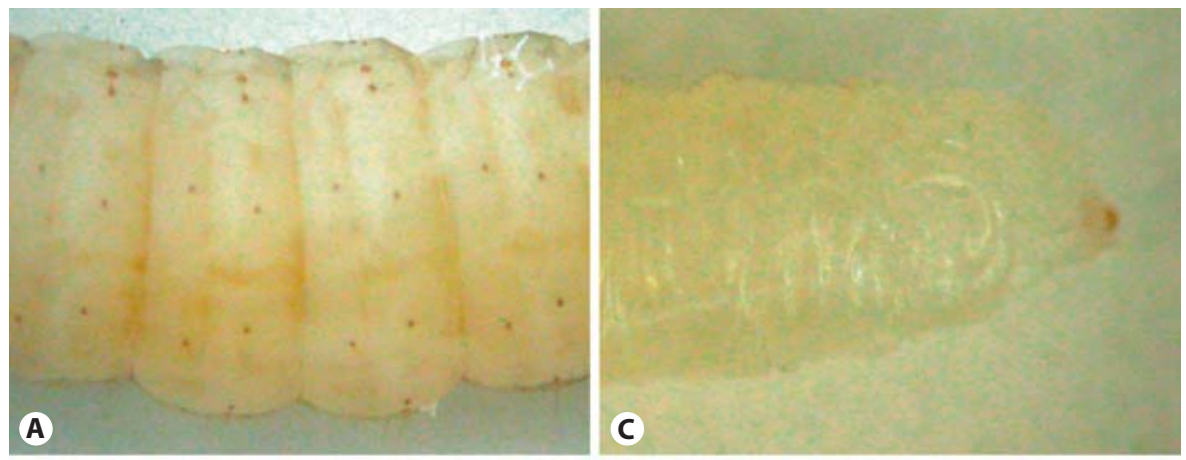

Fig. 1. Melanization in PGN-challenged larvae. Healthy Galleria (A, B) and Drosophila (C, D) last-instar larvae were injected with PGN and photographed $5 \mathrm{~min}(\mathbf{A}$, C) and $90 \mathrm{~min}(\mathbf{B}, \mathbf{D})$ after injection. The arrow in $\mathbf{D}$ indicates the injection site.
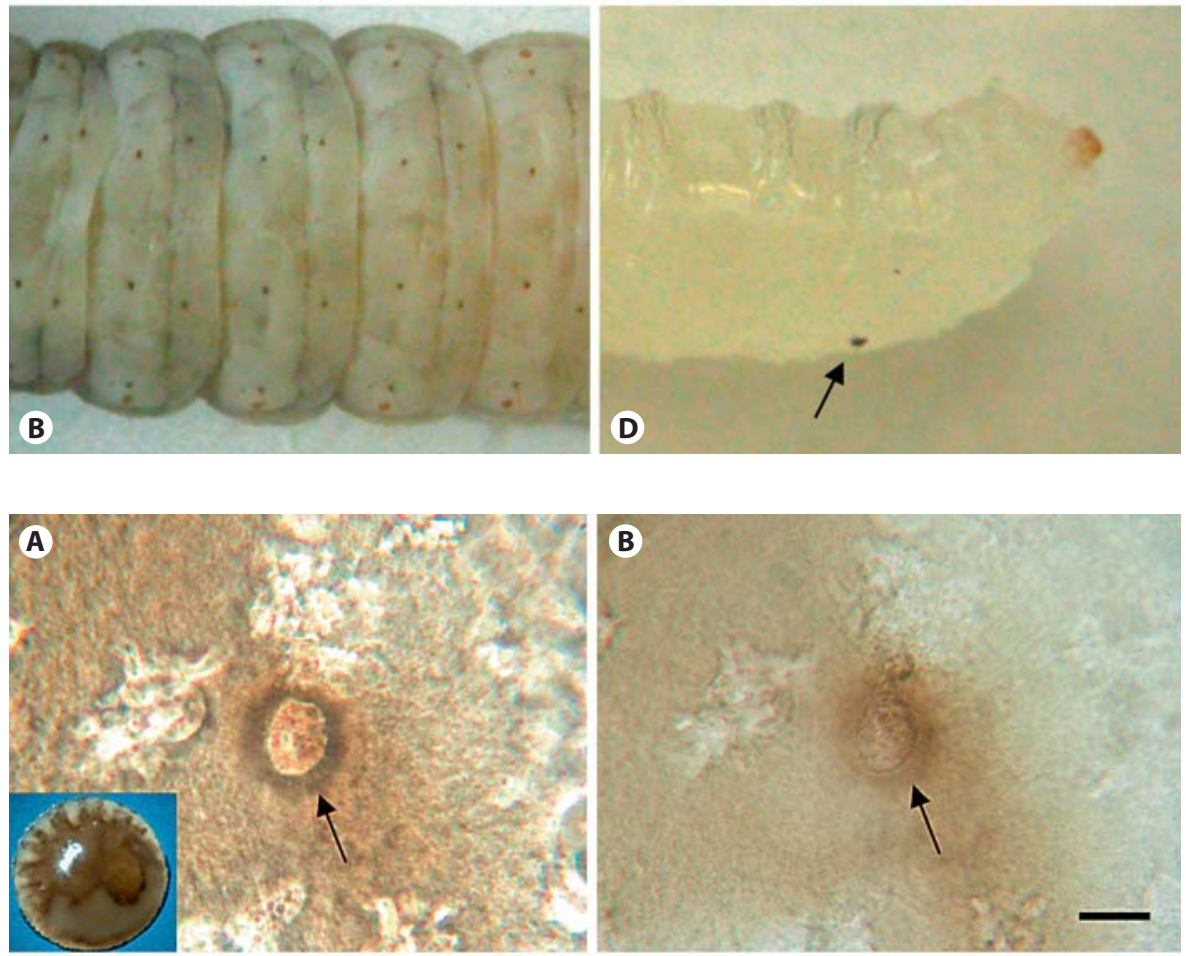

Fig. 2. Activation of PO in Galleria and Drosophila hemolymph clots. Hemolymph from Galleria (A, B) and Drosophila (C, D) were bled onto multiwell slides (a single well has a diameter of $4 \mathrm{~mm}$ ), allowed to melanize for $30 \mathrm{~min}$ and either photographed directly (insets in $\mathbf{A}$ and $\mathbf{C}$ ) or prepared using the hanging drop method and analyzed microscopically (A-D) using phase contrast $(\mathbf{A}, \mathbf{C})$ or transmission light (B, D). The arrows in $\mathbf{A}$ and $\mathbf{B}$ indicate a Galleria oenocytoid that has released PO. Scale bar $=10 \mu \mathrm{m}$.
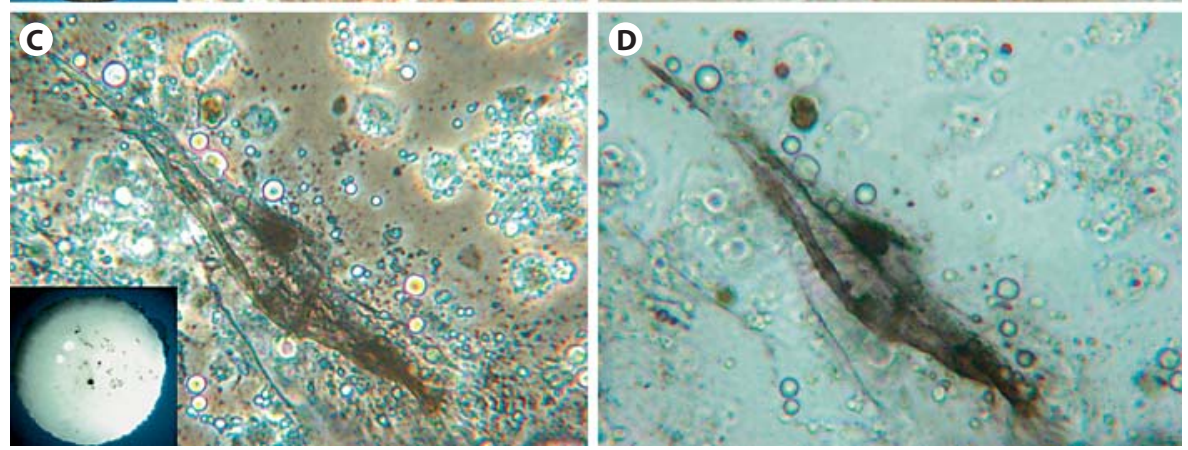

Drosophila larvae showed melanization only at the injection site (fig. 1C, D). To study the activation of proPO, larvae were bled and the clots that formed were examined. Melanization of hemolymph became visible with the naked eye after a few minutes. Galleria hemolymph melanized extensively (fig. 2A, inset), while comparable samples from Drosophila hemolymph remained almost transparent except for a few darker spots and strands (fig. 2C, inset). Microscopically, melanization of Galleria clot preparations was diffusely distributed over the en- 
tire sample (fig. 2A, B). In contrast, as expected from its macroscopic appearance, melanization in the Drosophila clot was highly localized and most intense along clot fibers and folds, as well as on cellular fragments derived from ruptured crystal cells or other hemocytes (fig. 2C, D) $[16]$.

\section{Drosophila proPO Is Activated after Injury by Endogenous Elicitors}

Our previous data suggested that in Drosophila, pro$\mathrm{PO}$ activation after injury involves signals released from ruptured crystal cells and other dying hemocytes [16]. This led us to speculate that inner-membrane lipids exposed during crystal cell rupture might activate proPO, similar to what happens during platelet activation in vertebrate blood clotting $[23,24]$. Supporting this idea, we found we could significantly superinduce melanization in ex vivo hemolmph preparations from noninfected an- imals using inner-membrane lipids [PS and phosphatidylinositol (PI); fig. 3A, B], which are hallmarks of damaged cells. These in vitro data are consistent with the melanization we observed after inducing apoptosis in hemocytes in vivo, which also leads to cell surface exposure of PS and subsequent formation of melanotic aggregates [16] (fig. 3D). Thus, proPO activation can also be induced in vivo in a restricted manner. Conversely, neither of the outer-membrane lipids (PC and PE) led to any significant increase in $\mathrm{PO}$ activity in a photometric assay (fig. 3A, B). The bacterial elicitors increased melanization in some cases (for example, for LPS in fig. 3A, and for PGN in some samples in fig. $3 \mathrm{~B}$ ), but in photometric assays, this effect was too variable to be significant. Melanization was completely inhibited when Spn27A was ubiquitously overexpressed (fig. 3B, 27A/OE), supporting our previous data from histological clot preparations [16].
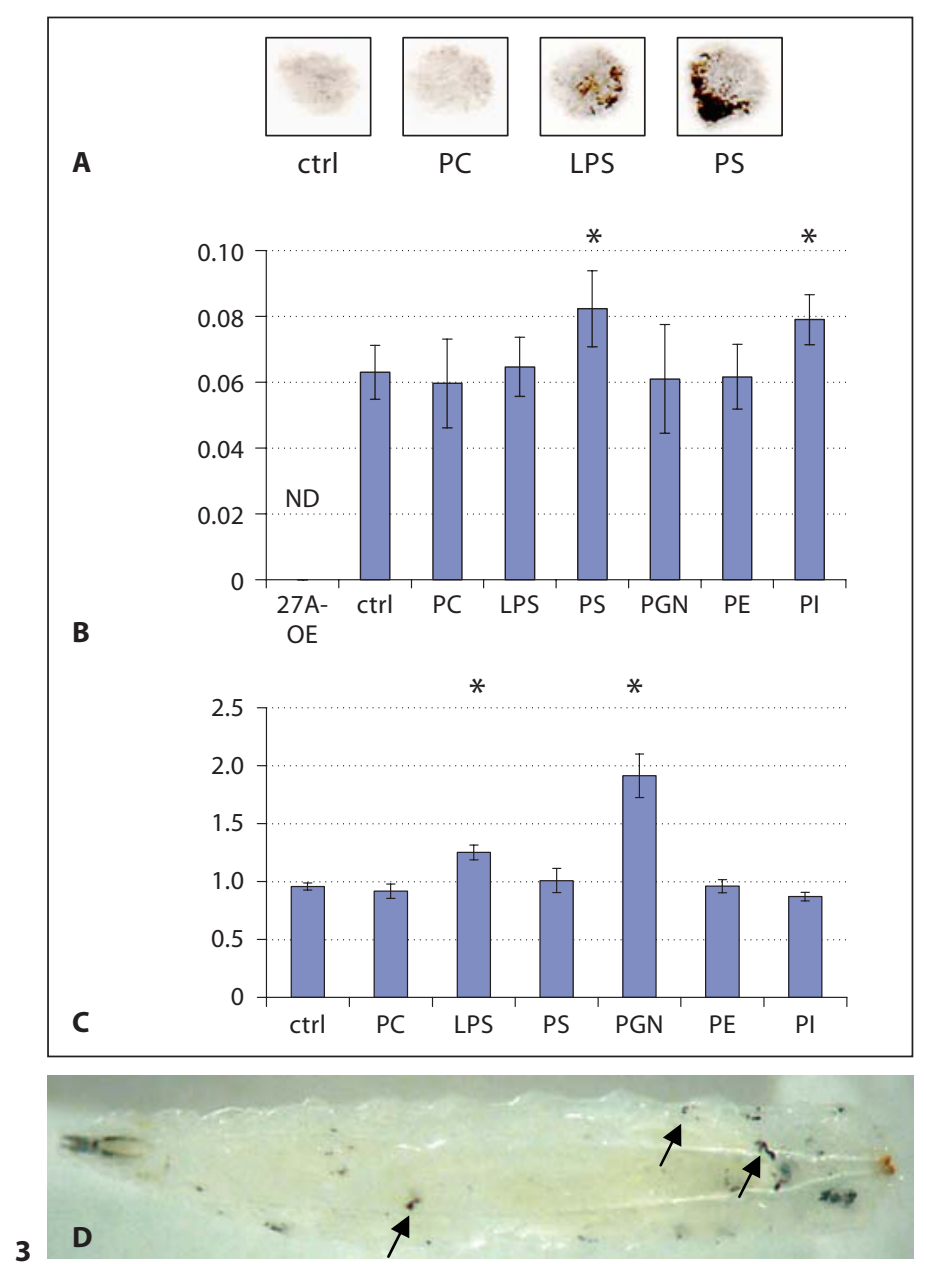

Fig. 3. PO activity is induced by endogenous signals. A The outermembrane lipid PC, bacterial LPS and the inner-membrane lipid PS were mixed with Drosophila hemolymph, and melanization was determined using a dot blot assay [36]. ctrl = Control sample with solvent alone. The experiment was repeated twice with the same result. B, C Hemolymph from Drosophila (B) and Galleria (C) was mixed with the same lipids as in $\mathbf{A}$ and in addition with bacterial PGN, PE and PI. Change of OD was measured as described (see Materials and Methods). Samples that differ significantly from the control are indicated by an asterisk. $\mathrm{p}$ values are: PS: 0.014; PI: 0.038; LPS: 0.0019; PGN: 0.00095. The Drosophila set was repeated in quadruplicate (see Materials and Methods for further details). When Spn27A was overexpressed ectopically using a tubulin driver line (27A-OE [16]), melanization was inhibited completely and was not detectable (ND). D Expression of the apoptotic inducer Grim in hemocytes (heGal4 crossed to UASgrim) leads to the formation of melanotic aggregates (some of which are indicated by arrows [see also 16]).

Fig. 4. proPO is not activated in clots from Spn27A mutants. Direct bleeds (A) and hanging drop preparations (B-I) from spn $27 \mathrm{~A}$ mutants (left) and control larvae (right) showing strong systemic activation in spn27A as described before $[4,5]$ (the insets in $\mathbf{D}$ and E show PO activity detected with the dot blot assay [36]). F-I Clot preparations from $w^{1118}(\mathbf{F}, \mathbf{G})$ and $\operatorname{spn} 27 A(\mathbf{H}, \mathbf{I})$ larvae were reconstituted with DOPA. All microscopic figures are shown in both phase-contrast (B, C, F, H) and transmission microscopy (D, E, G, I).

Fig. 5. Model of proPO activation in the clot. After release from crystal cells, $\mathrm{PO}$ is activated in a complex containing activating proteases and inner-membrane lipids released during crystal cell rupture. Inside the clot, proPO-activating complexes have limited contact with Spn27A, due to restricted diffusion of Spn27A into the clot matrix, while outside the clot, the proPO-activating proteases are inhibited. $\mathrm{nu}=$ Nucleus. 

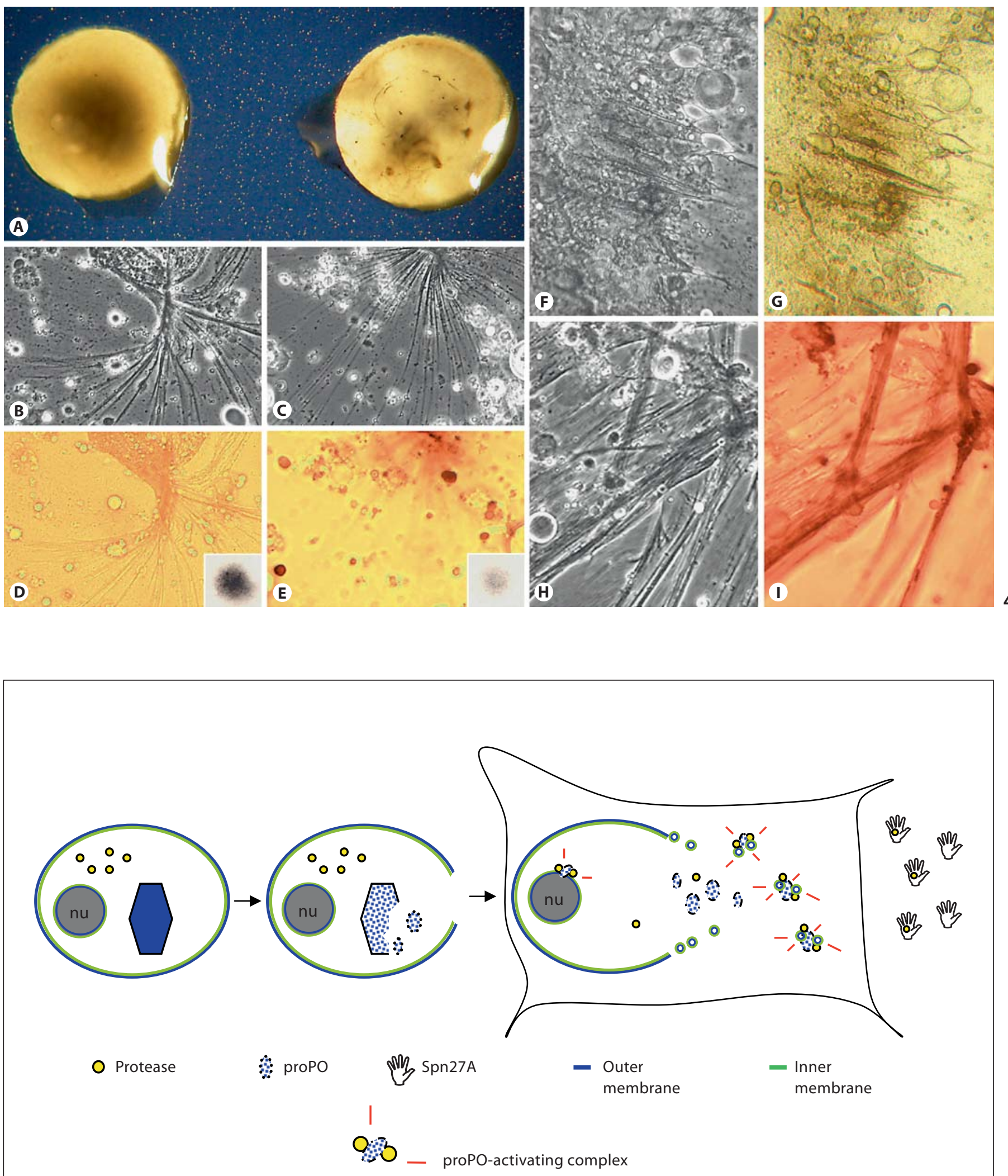

Activation of Insect Phenoloxidase after

J Innate Immun 2009;1:301-308 
The superactivation of proPO was different in Galleria. Here, both LPS and PGN significantly superinduced PO activity, while PS showed only marginal, nonsignificant effects, and neither PC nor PI had any effect (fig. 3C). This indicates that during activation of proPO after bleeding, endogenous signals such as inner-membrane lipids can act as activators in some species and not in others. In summary, proPO activation after injury appears to differ between Drosophila and Galleria; in Drosophila, activation is more locally restricted and can be enhanced by endogenous signals, while in Galleria, activation is more systemic and can be superinduced by microbial elicitors but not by endogenous lipids.

\section{proPO in the Clot Is Not Activated in Drosophila Spn27A Loss-of-Function Mutants}

Our results showed that in noninfected Drosophila larvae, $\mathrm{PO}$ activity is limited to the clot matrix, where it contributes to clot hardening and sequestration of bacteria [10]. We wished to relate these findings to previous studies on proPO activation in Drosophila that had been performed after septic injury. Following infection, Drosophila Spn27A is transiently degraded, allowing systemic activation of proPO (see Introduction). Spn27A loss-offunction mutants thus resemble infected animals and show high $\mathrm{PO}$ activity in their hemolymph $[4,5]$. Surprisingly, in spite of the absence of Spn27A and subsequent high systemic PO activity (fig. 4A, inset in fig. 4D), the clot from spn27A mutants showed no signs of melanization (fig. 4D). Similarly, clots from mutants expressing a dominant active form of Toll $\left(\mathrm{Tl}^{8}\right)$ mimicking immune activation during septic injury show no sign of melanization (not shown), in spite of the presence of melanotic masses in $\mathrm{Tl}^{8}$ hemolymph $[20,25]$. The lack of melanization in spn27A mutants appeared to be due to limited availability of $\mathrm{PO}$ substrate in the clot, since exogenous addition of DOPA restored clot melanization (fig. $4 \mathrm{H}, \mathrm{I}$ ) to a level which was comparable to or even stronger than that in normal animals treated identically (fig. $4 \mathrm{~F}, \mathrm{G}$ ). Hemolymph preparations from spn27A mutants looked similar to those from Galleria, with high systemic PO activity and weak $\mathrm{PO}$ activity in the clot (compare fig. 2 and 4).

\section{Discussion}

We have incorporated our findings into a model for proPO activation in Drosophila (fig. 5). Based on our finding that melanization in the clot can be inhibited by
spn27A (fig. 3B) [16], we propose that clot proPO is activated proteolytically. Following wounding, crystal cells are activated and rupture [16] and proPO is released and forms complexes with activating proteases and aminophospolipids such as PS that are also released during cell rupture. Association with activating proteases may be facilitated by the fact that some of them are expressed in crystal cells, for example CG3066 (or MP2 [6, 8]). Association with lipids may facilitate proteolytic cleavage, in agreement with previous findings that slightly denaturing conditions can activate proPO [summarized in 26]. We propose that normally proPO activation is limited to the clot matrix by Spn27A, which has limited access to the clot but inhibits any proPO activation outside the clot. In many cases, the formation of a clot matrix will be sufficient to sequester any microbes that have entered via the wound, thus preventing sepsis. In case bacterial entrapment by the clot is insufficient and results in bacteremia, microbial products trigger the transcriptional activation of immune genes and activate a humoral response, including breakdown of Spn27A and systemic activation of proPO $[4,5]$.

Thus, in Drosophila, one and the same immune reaction, i.e. melanization, is activated differently at different times and places following injury. Endogenous triggers such as lipids (this report) and basement membrane components released during injury [summarized in 27, 28] activate proPO at the clot soon after wounding, contributing to crosslinking and melanization independent of bacterial elicitors. This is somewhat unexpected since microbial breakdown products are likely present in high concentrations in decaying fruits, where Drosophila larvae usually feed. In the case of a septic infection, however, it has been shown that microbial products do cause systemic gene transcription-dependent proPO activation $[4,5]$.

The situation appears to be different in Galleria, where proPO activation at the preseptic stage is more systemic and sensitive to microbial elicitors before the onset of any transcriptional activation (fig. 1A, B, 2A, B, 3C). A possible molecular mechanism to explain the systemic melanization is suggested by the similarity of clots from $\mathrm{Gal}$ leria and Drosophila spn27A mutants. According to our model (fig. 5), the different mode of activation of Galleria proPO might be due to a lower concentration of inhibitory activities such as Spn27A allowing activation of proPO even outside the clot matrix. In addition, the availability of PO substrates may influence its activity [29] (fig. 4). 
At this stage, we can only speculate on the environmental and evolutionary factors that determine how pro$\mathrm{PO}$ is activated in different animals. It may be that more localized and endogenously activated melanization ensures fast and efficient crosslinking of clot fibers in small animals like Drosophila and mosquitoes, where clot melanization appears similar [10, 14, Hauling et al., unpubl. results]. Reliance on endogenous signals may on the other hand enhance the risk of autoimmune reactions. This appears to happen in spn27A mutants, where the prevalence of spontaneous melanization increases, leading to a reduction in viability [5].

Our results add new findings to the ongoing discussion regarding the role of $\mathrm{PO}$ in innate immunity. It is interesting to note that most of the recent reports arguing against an immune function involve Drosophila or mosquitoes, where our data suggest $\mathrm{PO}$ is regulated by endogenous signals and contributes primarily to hemostasis at least at the wound. In contrast, we found that microbial elicitors were more effective at activating proPO in
Galleria, a lepidopteran. Here, the interference with pro$\mathrm{PO}$ activation caused by virulence factors isolated from parasitoid wasps or bacterial commensals from parasitic nematodes of lepidopterans [30-32] is strong evidence for an immune function of PO.

Taken together, our data suggest that PO activity is used in different ways depending on physiological and ecological requirements. This may caution against generalizations based on studies performed in one particular insect species or with one particular pathogen, and is in complete agreement with recent studies based on genomic comparisons which show faster evolution for factors that regulate $\mathrm{PO}$ activity $[2,33-35]$.

\section{Acknowledgments}

This work was supported by research grants from the Swedish Research Council, (U.T. and M.S.D.), Stockholm University (U.T.) and Södertörns Högskola (M.S.D.). We thank the reviewers for constructive feedback.

\section{References}

1 Nappi AJ, Ottaviani E: Cytotoxicity and cytotoxic molecules in invertebrates. Bioessays 2000;22:469-480.

-2 Cerenius L, Lee BL, Söderhäll K: The proPOsystem: pros and cons for its role in invertebrate immunity. Trends Immunol 2008;29: 263-271.

3 Kanost MR, Gorman MJ: Phenoloxidases in insect immunity; in Beckage NE (ed): Insect Immunology. Amsterdam, Elsevier, 2008, pp 69-96.

4 De Gregorio E, Han SJ, Lee WJ, et al: An immune-responsive Serpin regulates the melanization cascade in Drosophila. Dev Cell 2002;3:581-592.

5 Ligoxygakis P, Pelte N, Ji C, et al: A serpin mutant links Toll activation to melanization in the host defence of Drosophila. ЕMBO J 2002;21:6330-6337.

6 Castillejo-Lopez C, Häcker U: The serine protease Sp7 is expressed in blood cells and regulates the melanization reaction in Drosophila. Biochem Biophys Res Commun 2005;338:1075-1082.

7 Leclerc V, Pelte N, El Chamy L, et al: Prophenoloxidase activation is not required for survival to microbial infections in Drosophila. EMBO Rep 2006;7:231-235.

-8 Tang H, Kambris Z, Lemaitre B, Hashimoto C: Two proteases defining a melanization cascade in the immune system of Drosophila. J Biol Chem 2006;281:28097-28104.
-9 Medzhitov R, Janeway CA Jr: Decoding the patterns of self and nonself by the innate immune system. Science 2002;296:298-300.

10 Bidla G, Lindgren M, Theopold U, Dushay MS: Hemolymph coagulation and phenoloxidase in Drosophila larvae. Dev Comp Immunol 2005;29:669-679.

11 Shiao SH, Whitten MM, Zachary D, Hoffmann JA, Levashina EA: Fz2 and cdc42 mediate melanization and actin polymerization but are dispensable for Plasmodium killing in the mosquito midgut. PLoS Pathog 2006; 2:e133.

12 Schnitger AK, Kafatos FC, Osta MA: The melanization reaction is not required for survival of Anopheles gambiae mosquitoes after bacterial infections. J Biol Chem 2007; 282:21884-21888.

$\checkmark 13$ Li D, Scherfer C, Korayem AM, Zhao Z, Schmidt O, Theopold U: Insect hemolymph clotting: evidence for interaction between the coagulation system and the prophenoloxidase activating cascade. Insect Biochem Mol Biol 2002;32:919-928.

14 Agianian B, Lesch C, Loseva O, Dushay MS: Preliminary characterization of hemolymph coagulation in Anopheles gambiae larvae. Dev Comp Immunol 2007;31:879-888.

15 Scherfer C, Karlsson C, Loseva O, et al: Isolation and characterization of hemolymph clotting factors in Drosophila melanogaster by a pullout method. Curr Biol 2004;14:625629.
16 Bidla G, Dushay MS, Theopold U: Crystal cell rupture after injury in Drosophila requires the JNK pathway, small GTPases and the TNF homolog Eiger. J Cell Sci 2007;120: 1209-1215.

17 Arakane Y, Muthukrishnan S, Beeman RW, Kanost MR, Kramer KJ: Laccase 2 is the phenoloxidase gene required for beetle cuticle tanning. Proc Natl Acad Sci USA 2005;102: 11337-11342.

18 Rowley AF, Ratcliffe NA: The granular cells of Galleria mellonella during clotting and phagocytic reactions in vitro. Tissue Cell 1976;8:437-446.

19 Galko MJ, Krasnow MA: Cellular and genetic analysis of wound healing in Drosophila larvae. PLoS Biol 2004;2:E239.

20 Zettervall CJ, Anderl I, Williams MJ, et al: A directed screen for genes involved in Drosophila blood cell activation. Proc Natl Acad Sci USA 2004;101:14192-14197.

21 Wing JP, Schwartz LM, Nambu JR: The RHG motifs of Drosophila Reaper and Grim are important for their distinct cell death-inducing abilities. Mech Dev 2001;102:193-203.

22 Majumder R, Wang J, Lentz BR: Effects of water soluble phosphotidylserine on bovine factor Xa: functional and structural changes plus dimerization. Biophys J 2003;84:12381251.

23 Barry OP, FitzGerald GA: Mechanisms of cellular activation by platelet microparticles. Thromb Haemost 1999;82:794-800. 
-24 Horstman LL, Ahn YS: Platelet microparticles: a wide-angle perspective. Crit Rev Oncol Hematol 1999;30:111-142.

-25 Schneider DS, Hudson KL, Lin TY, Anderson $\mathrm{KV}$ : Dominant and recessive mutations define functional domains of Toll, a transmembrane protein required for dorsal-ventral polarity in the Drosophila embryo. Genes Dev 1991;5:797-807.

-26 Ashida M: The prophenoloxidase cascade in insect immunity. Res Immunol 1990;141: 908-910.

-27 Schmidt O, Theopold U, Strand M: Innate immunity and its evasion and suppression by hymenopteran endoparasitoids. Bioessays 2001;23:344-351.

-28 Brennan CA, Anderson KV: Drosophila: the genetics of innate immune recognition and response. Annu Rev Immunol 2004;22:457483.
29 Gorman MJ, An C, Kanost MR: Characterization of tyrosine hydroxylase from Manduca sexta. Insect Biochem Mol Biol 2007;37: 1327-1337.

30 Beck M, Theopold U, Schmidt O: Evidence for serine protease inhibitor activity in the ovarian calyx fluid of the endoparasitoid Venturia canescens. J Insect Physiol 2000;46: 1275-1283.

31 Eleftherianos I, Boundy S, Joyce SA, et al: An antibiotic produced by an insect-pathogenic bacterium suppresses host defenses through phenoloxidase inhibition. Proc Natl Acad Sci USA 2007;104:2419-2424.
2 Beck MH, Strand MR: A novel polydnavirus protein inhibits the insect prophenoloxidase activation pathway. Proc Natl Acad Sci USA 2007;104:19267-19272.

33 Waterhouse RM, Kriventseva EV, Meister S, et al: Evolutionary dynamics of immune-related genes and pathways in disease-vector mosquitoes. Science 2007;316:1738-1743.

>34 Clark AG, Eisen MB, Smith DR, et al: Evolution of genes and genomes on the Drosophila phylogeny. Nature 2007;450:203-218.

35 Sackton TB, Lazzaro BP, Schlenke TA, Evans JD, Hultmark D, Clark AG: Dynamic evolution of the innate immune system in Drosophila. Nat Genet 2007;39:1461-1468.

36 Sorrentino RP, Small CN, Govind S: Quantitative analysis of phenol oxidase activity in insect hemolymph. Biotechniques 2002;32: 815-816, 818, 820, 822-823. 